\title{
Self-Pruning based Probabilistic ApProach TO MINIMIZE REDUNDANCY OVERHEAD FOR PERFORMANCE IMPROVEMENT IN MANET
}

\author{
Gyanendra Kumar Pallai ${ }^{1}$, Meenakshi Sankaran ${ }^{2}$ and Amiya Kumar Rath ${ }^{3}$ \\ ${ }^{1}$ Department of Computer Science \& Engineering, \\ SOA Deemed-to-be University, Bhubaneswar, India, \\ ${ }^{2}$ VIT Business School, VIT Bhopal University, Bhopal, India \\ ${ }^{3}$ Department of Computer Science \& Engineering, Veer Surendra Sai University of \\ Technology, Burla. Deputed as adviser to National Assessment \& Accreditation \\ Council, Bengaluru, India
}

\begin{abstract}
The Broadcast storm problem causes severe interference, intense collision and channel contention, which greatly degrades the QoS performance metrics of the routing protocols. So, we suggest a neighbourhood coverage knowledge probabilistic broadcasting model (NCKPB) integrating with AODV protocol with knowledge on 2-hop neighbourhood coverage; a connectivity function to control a node's forwarding probability of retransmission to alleviate significant overhead redundancy. Our objective is to minimize the broadcast RREQ overhead while ensuring fair retransmission bandwidth. We considered two more important measures called Saved Rebroadcast and Reachability. The outcomes of NCKPB, Fixed probability $(F P)$ and Flooding (FL) routing schemes are examined under three major operating conditions, such as node density, mobility and traffic load. The NS-2 results demonstrate the efficacy of the proposed NCKPB model by illustrating its performance superiority over all key metrics such as redundancy overhead, end to end latency, throughput, reachability, saved rebroadcast and collision contrast to FP and FL.
\end{abstract}

\section{KEYWORDS}

Broadcasting, Flooding, Self-pruning, Neighbour Coverage, Forwarding Probability, Connectivity Metric.

\section{INTRODUCTION}

MANET is a dynamically self-organized network of freely moving nodes without any fixed infrastructure [1] [2]. Arbitrary node mobility in ad hoc networks leads to regular errors in synchronisation among source and destination nodes, thus growing the number of redundancy overheads that degrade the efficiency of the routing protocol. Broadcasting [10] is a typical method in MANET for exploring paths. In this method each node sends a RREQ message among its neighbouring nodes within the transmitting range. Thus the RREQ message passes through intermediary nodes across the network in multi-hop mode. A traditional flooding scheme [10] [11] [12] enforces broadcasting, wherein each node retransmit a RREQ message upon receiving the broadcast message for the first time. Flooding executes at every node to ensure the message must reach to every node in the network. This results in severe interference, excessive collisions and channel contention within mobile wireless networks, known as the "broadcast storm problem" [10] [11] [12]. Channel contention [10] happens when a node $X$ retransmits a message it receives from node $\mathrm{Y}$ when another node $\mathrm{Z}$ tries to retransmit at the same time. Then node $\mathrm{Z}$ needs to wait for a random amount of time, since it presumes that the channel is busy due to node $\mathrm{Y}$. Node $\mathrm{X}$ will 
compete substantially with node Y's common shared link, which increases the data transmission latency time. Message collision [10] or overlapping node transmission is more likely to occur because there is no detection process in the simple flooding scheme. Say node X and Y, for example, attempt to retransmit the message simultaneously results in collisions for which the message could not reach any other node say $\mathrm{Z}$.

Basically broadcasting mechanism for route discovery process in MANET reactive protocols are conducted via blind flooding. The core issue is how to reduce the amount of rebroadcasts thereby preserving reasonable latency for retransmission and reachability of packets. We understand that a huge volume of duplicate rebroadcasts imply a high degree of reachability, but at the same time they degrade the efficiency of the protocol due to bandwidth wastage and collisions. Conversely, limited amount of retransmission results in poor reachability since it causes the retransmission chain to be split in such a way that certain hosts are unable to receive the packets sent. Hence, how to lessen the amount of rebroadcasts whilst retaining fair bandwidth, overhead and packet reachability is a significant issue.

This research paper discusses our proposed scheme integrating with AODV protocol using neighbour knowledge information (NCKPB). To reduce unnecessary retransmissions, our method blends the benefits of probabilistic and neighbourhood information with a connectivity metric. The rest of this study is arranged as follows: We discuss the relevant studies on MANET broadcasting on Section 2. We discuss the MANET routing protocols and route discovery process of AODV protocol in Section 3. Section 4 reviews detail of our proposed broadcast scheme NCKPB on AODV and its algorithm. In Section 5 we simulate our method using NS-2 [9] simulator. The results of the simulation and contrast its output with FL and FP. In section 6, we summarize the paper and identify approaches for future investigation.

\section{RELATED WORK}

Numerous broadcasting solutions have been introduced in recent times to optimize the MANETs' retransmission techniques. The B.Williams and Camp [13] description of broadcasting systems is based on probability, area and neighbourhood knowledge. Basic flooding [3] [10] [12] [13] is a simplistic broadcasting solution which is quick to enforce with assured transmission of messages. Every host rebroadcasts with a predetermined probability $\mathrm{P}(\mathrm{P}=1)$ [19]. Simplistic FL offers adequate transmission but often causes redundancy, contention, and collision. MANET has suggested several probabilistic [14] [16] [17] [18] [19] [20] solutions as a way to reduce redundant transmission and mitigate the adverse impact of the issue of broadcast storm. Fixed probabilistic [10] [15] is the most basic probabilistic strategy, from the basis of which all subsequent dynamic probabilistic schemes are defined. In FP, the probability $\mathrm{P}$ of each host is generally assumed to be between 0.65 and 0.7. M.Bani-Yassein et.al. [17] performed a detailed ns-2 simulation to research the efficiency of optimised FP and flooding strategy. A technique that combines the dynamic probabilistic and fixed probabilistic approach to decrease the RREQ overhead for different node speed has been implemented in the work [20]. The probability of forwarding is dynamically regulated by each node, based on their neighbourhood density level in the network has been studied in [18] [19]. A mixture of probabilistic and counter-based method was suggested by Qi Zhang et.al.[16] contrasted with flooding and set probabilistic schemes for different network conditions such as network size, speed and CBR connections. The suggested DP-AODV solution yields less retransmissions, greater reachability, higher throughput and minimal latency by dynamically regulating a node's probability of forwarding utilising its neighbouring density. Jae-soo kim et.al. [23] presents a broadcast technique that incorporates a probabilistic and area-based method. The receiver node calculates the additional range by estimating the distance from the sender using signal intensity and dynamically controls the forwarding probability. In flooding with self-pruning [21] proposed by Lim and Kim, the broadcasting node appends its one-hop neighbours to the RREQ 
packet. The receiver node matches the list of neighbours from the received RREQ message with its own surrounded neighbour. W. Peng et.al [22] suggested a scalable broadcast algorithm (SBA) focused on neighbour information within a distance of two hops. Xin Zhang Ming et al. [24] developed a probabilistic rebroadcast protocol (NCPR) for neighbourhood coverage. The scheme used a balanced rebroadcast delay to estimate neighbourhood information along with additional neighbour coverage and a connectivity function. A neighbour based routing protocol DCFP [25] was developed and tested against NCPR and AODV protocols to minimise the redundant RREQ packets. DCFP dynamically investigates the neighbourhood knowledge using novel network connectivity metric and preset parameters. An enhanced self-pruning broadcasting (ISB) algorithm [26] is suggested, and its output is evaluated with dominant and other self-pruning schemes under different transmission ranges utilising three-hop neighbour knowledge information. Huaqiang Xu et.al [29] explored a probabilistic broadcast scheme TPB centred on nodes trust level. Dingzhu Lu et.al.[27] has introduced a two neighbour knowledge based and velocity based schemes namely NKB and NKVB. The efficiency of two systems is measured against other current broadcasting structures for differing node size, maximum speed and CBR load. A density-aware probabilistic strategy to alleviate broadcast storm effects for content-centric vehicular networks have been suggested in the work [28] and its performance is compared with other related approaches.

\section{Routing Protocols in MANet}

In order to overcome routing challenges in MANETs, many routing protocols [2] have recently been studied. These protocols were categorised under three strategically defined routing classifications [2] [3]: proactive, reactive, and hybrid. Table-driven routing protocols [5] [30] [31] are considered proactive protocols, constantly keeping the updated information for whole network in a routing table (RT). The greatest benefit of proactive protocols is that with minimal end-to - end delay it transmits data immediately. However, Periodic update of the routing table (RT) raises the overhead as compared to the reactive protocol. The Destination Sequence Distance Vector (DSDV) and Optimized link state Routing (OLSR) is of proactive category. The reactive ones [3] [4] [7] [30] [31] are an enhancement to table-driven routing that uses the method of route discovery only when the source node desires it. Unlike proactive protocols, reactive protocols only hold knowledge regarding active paths. Compared with the proactive category, this results in less redundancy overhead. Ad hoc On-Demand Distance Vector Routing (AODV) and Dynamic Source Routing (DSR) are two basic reactive MANET protocols. The Hybrid [6] incorporates the scalability benefit of the reactive protocol plus proactive protocol performance. For instance, Zone Routing Protocol. Hybrid protocols split their neighbouring nodes into two domains. One called the "intra-zone" and uses reactive protocol, while the other designated "inter-zone" investigates by proactive protocol.

\subsection{Route Discovery Mechanism in AODV}

AODV has gained a considerable interest owing to its simplicity and low processing overhead. In this scheme, a fresh path is established by a source node on demand. Unlike proactive, it does not update its routing table (RT) periodically and thus incurs less control overhead. Any source node has some packets to deliver to its destination, it first searches the path availability with its own routing table (RT) [3] [7]. If the path is available in the RT, it literally follows the path to deliver the message otherwise it invokes route discovery mechanism [3] [7]. First, the source broadcasts the RREQ notification to all of its neighbourhood nodes. Any mobile host that receives the broadcast message increases its hop count and builds a reversed route (for source and neighbour node) with an update to RT. A unicast RREP message is sent to source either from destination or any node that has fresh path information; otherwise the node propagates the RREP notification to all the neighbours around it. The node receives an RREP message, it immediately generates the forward route entry for the node (i,e. destination or intermediary node) RREP message was made. Using the 
reverse path entry, the node passes the RREP message until it reached to source node. If the source node obtains multiple RREPs along separate paths, the path with the highest destination sequence number is selected.

\section{Proposed Scheme - Neighbour Coverage Knowledge Probabilistic Broadcasting Protocol (NCKPB)}

The main purpose and motivation of our proposed scheme is to find a way of alleviating broadcast storm problems that cause extreme collision and channel contention and optimise the performance of routing protocols across all performance metrics. The suggested methodology is a reactive approach to self-pruning broadcasting. A mobile host makes a decision on rebroadcast using 2-hop neighbourhood knowledge with redundant information to minimize the redundancy overhead. For explicit exploration of neighbourhood knowledge, every host shares "hello" packets periodically. If the estimated time interval for rebroadcasting is short, it contributes to excessive collision or contention. Moreover, if the neighbouring nodes of a node have been covered by previous broadcasts, a node should not retransmit the packet.

The suggested NCKPB broadcast strategy is structured in four steps:

- Estimation of a reasonable rebroadcast delay for a node.

- Acquisition of neighbouring information to determine additional coverage area.

- A connectivity function to balance the amount of retransmissions.

- Regulating the probability of retransmissions based on the additional neighbour coverage fraction with connectivity function.

\subsection{Rebroadcast Delay}

In order to optimise the neighbourhood coverage of the node effectively, it is important to identify a reasonable delay in rebroadcasting. A node will be given a lower retransmission if there were a large amount of similar neighbours instead we would set a higher limit.

The delay ratio $\mathrm{T}_{\mathrm{d} \text {-ratio }}$ of $\mathrm{n}_{\mathrm{r}}$ is calculated is as follows:

$$
T_{\text {d-ratio }}\left(n_{r}\right)=1-\frac{N\left(n_{5}\right) \cap N\left(n_{r}\right)}{N\left(n_{5}\right)}
$$

Rebroadcast delay Trb-delay is estimated using max delay constant " $\Delta^{w}$, we choosen $0.1 \mathrm{sec}$.

$$
\mathbf{T}_{\text {rb-delay }}\left(\mathbf{n}_{\mathbf{r}}\right)=\Delta \times \mathbf{T}_{\text {d-ratio }}\left(\mathbf{n}_{\mathbf{r}}\right)
$$

\subsection{Additional Coverage Ratio}

A receiver node $\mathrm{nr}$ accepts an RREQ from sender ns , the recipient nr compares the sender list to its own neighbours. The receiving node nr would then compile an uncovered list of neighbours. The prepared list contains all neighbour nodes of the receiver not covered by the sender node. This uncovered neighbour list gives an approximation for estimating the extra coverage ratio of the receiver. This means, if the receiving node $\mathrm{nr}$ rebroadcasts the packet, it will reach more adjacent nodes. 
International Journal of Computer Networks \& Communications (IJCNC) Vol.13, No.2, March 2021

The additional coverage ratio $\mathrm{A}_{\text {cov-ratio }}$ of $\mathrm{n}_{\mathrm{r}}$ is as follows:

$$
\mathbf{A}_{\text {cov-ratio }}\left(\mathbf{n}_{\mathbf{r}}\right)=\frac{\mathbf{N}\left(\mathbf{n}_{\mathbf{r}}\right)-\mathbf{N}\left(\mathbf{n}_{\mathbf{s}}\right) \cap \mathbf{N}\left(\mathbf{n}_{\mathbf{r}}\right)}{\mathbf{N}\left(\mathbf{n}_{\mathbf{r}}\right)}
$$

\subsection{Connectivity Metric}

We evaluate a connectivity metric $\mathrm{C}_{\text {metric }}$ on basis of average amount of neighbours in the network to control the quantity of RREQ retransmissions and computed as:

$$
\operatorname{Navg-nb}(\mathbf{n r})=\frac{(\mathrm{N}-1) \mathbf{K} \pi \mathrm{R}^{2}}{\text { Area }}
$$

$\mathrm{N}$ - Total number of network nodes, $\mathrm{R}$ - Transmission radius, A - Topology size, and

$\mathrm{K}$ - Connectivity constant (1.02).

$$
C_{\text {metric }}\left(n_{r}\right)=\frac{N_{\text {avg-nb }}\left(n_{r}\right)}{N\left(n_{r}\right)}
$$

\subsection{Forwarding Probability}

The forwarding probability $\mathrm{P}_{\mathrm{fw}}$ is a product of connectivity metric $\mathrm{C}_{\text {metric }}$ with additional coverage ratio $\mathbf{A}_{\text {cov-ratio and calulated as: }}$

$\mathbf{P}_{\mathbf{f w}}\left(\mathbf{n}_{\mathbf{r}}\right)=\mathbf{C}_{\text {metric }}\left(\mathbf{n}_{\mathbf{r}}\right) \times \mathbf{A}_{\text {cov-ratio }}\left(\mathbf{n}_{\mathbf{r}}\right)$

Measurement of the forwarding probability $\mathrm{P}_{\mathrm{fw}}$ with a random value (0 to 1$)$ provides knowledge of the node's neighbour density (ie. dense or sparse). If the node's forwarding probability is lower than the random value, this signifies that node is found in dense region. To minimize number of redundancy overhead, we must discard duplicate RREQ messages. However, if forwarding probability is bigger than random value, then it is found in the sparse zone and the message needs to be rebroadcasted.

Steps involved in our proposed scheme:

1. Each node sends its recent neighbouring list in the RREQ message. Upon receiving themessage from sender $\mathrm{n}_{\mathrm{S}}$, the receiver $\mathrm{nr}$ compares the sender list to its own neighbours list.

If $\mathbf{N}\left(\mathbf{n}_{\mathbf{r}}\right) \subseteq\left[\mathbf{N}\left(\mathbf{n}_{\mathbf{S}}\right) \cup\left\{\mathbf{n}_{\mathbf{S}}\right\}\right]$ Cancel Rebroadcast (Duplicate msg)

2. Otherwise, RREQ msg is received for the first time, $\mathrm{nr}$ prepares a covered neighbour set

$$
\text { Bcov-set }\left(\mathbf{n}_{\mathbf{r}}, \mathbf{m s g}\right)=\mathbf{N}\left(\mathbf{n}_{\mathbf{s}}\right) \cup\left\{\mathbf{n}_{\mathbf{s}}\right\}
$$

3. Rebroadcast delay Trb_delay using equation (2) for $\mathrm{nr}$ is calculated.

4. Node $\mathrm{nr}$ updates the covered neighbour set for each msg and discards the duplicate

$$
\text { Bcov-set (nr, msg) = Bcov-set (nr, msg) } \cup\left[\mathbf{N}\left(\mathbf{n}_{\mathbf{s}}\right) \cup\left\{\mathbf{n}_{\mathbf{S}}\right\}\right]
$$

5. Rebroadcast decision is made after the delay expires; If the Bcov-set ( $\mathrm{nr}, \mathrm{msg}$ ) covers all theneighbours of $\mathrm{nr}$, then the receiver nr not to rebroadcast

If $\mathbf{N}(\mathbf{n r}) \subseteq$ Bcov-set (nr, msg) Cancel rebroadcast

6. Otherwise, additional coverage ratio Acov-ratio (nr) is computed using eq (3).

7. Neighbour density Navg-nb and connectivity metric Cmetric estimated using eq (4) and (5). 
International Journal of Computer Networks \& Communications (IJCNC) Vol.13, No.2, March 2021

8. Forwarding probability $\mathbf{P f w}$ of $\mathrm{nr}$ is evaluated using eq (6).

9. Finally, the rebroadcast probability $\mathbf{P} \mathbf{f w}$ is compared against random value ranging between 0 and 1 for determining RREQ retransmissions.

\subsection{Algorithm - Neighbour Knowledge Probabilistic Broadcasting (NCKPB)}

Definitions: msg - RREQ message, $\mathrm{N}\left(\mathrm{n}_{\mathrm{r}}\right)$ - Neighbour set of $\mathrm{n}_{\mathrm{r}}, \mathrm{N}\left(\mathrm{n}_{\mathrm{s}}\right)$ - Neighbour set of $n_{s}$, Bcov-set(nr, msg) - Broadcast cover set of node $n_{r}$ for msg.

1. When a node $\mathrm{n}_{\mathrm{r}}$ receives a RREQ msg from $\mathrm{n}_{\mathrm{S}}$ :

2. If $\mathbf{N}\left(\mathbf{n}_{\mathbf{r}}\right) \subseteq\left[\mathbf{N}\left(\mathbf{n}_{\mathbf{s}}\right) \cup\left\{\mathbf{n}_{\mathbf{S}}\right\}\right]$ Cancel Rebroadcast

3. If (RREQ msg received for the first time)

4. Bcov-set $(\mathbf{n r}, \mathbf{m s g})=\mathbf{N}\left(\mathbf{n s}_{\mathbf{s}}\right) \cup\left\{\mathbf{n}_{\mathbf{s}}\right\}$

5. Calculate Rebroadcast delay:

6. Td-ratio $\left(n_{r}\right)=1-\frac{N\left(n_{s}\right) \cap N\left(n_{r}\right)}{N\left(n_{s}\right)}$

7. $\mathbf{T r b}-\operatorname{delay}(\mathbf{n r})=\Delta \mathbf{x} \mathbf{T}$ d-ratio $(\mathbf{n r})$

8. Schedule the rebroadcast timer for $\operatorname{Trb}$-delay $(\mathrm{nr})$

9. End if

10. While ( $\mathrm{n}$ receives duplicate msg during the timer period)

11. Update the broadcast cover set:

12. $\mathbf{B}$ cov-set $\left(\mathbf{n}_{\mathbf{r}}, \mathbf{m s g}\right)=\mathbf{B} \operatorname{cov}-\operatorname{set}\left(\mathbf{n}_{\mathbf{r}}, \mathbf{m s g}\right) \cup\left[\mathbf{N}\left(\mathbf{n}_{\mathbf{s}}\right) \cup\left\{\mathbf{n}_{\mathbf{s}}\right\}\right]$

13. Discard msg

14. End while

15. If (Timer for rebroadcasting node $n_{r}$ expires)

16. If $\mathbf{N}\left(\mathbf{n}_{\mathbf{r}}\right) \subseteq \mathbf{B}$ cov-set(nr, $\left.\mathbf{m s g}\right)$ Cancel rebroadcast

17. Else

18. Calculate the coverage ratio:

19. Acov-ratio $\left(n_{r}\right)=\frac{N\left(n_{r}\right)-N\left(n_{s}\right) \cap N\left(n_{r}\right)}{N\left(n_{r}\right)}$

20. Calculate average network neighbour density:

21. $\operatorname{Navg-nb}\left(n_{r}\right)=\frac{(N-1) K \pi R^{2}}{\text { Area }}$

22. Calculate connectivity factor:

23. $C_{\text {metric }}\left(\mathbf{n}_{\mathbf{r}}\right)=\frac{\mathbf{N}_{\text {avg-nb }}\left(\mathbf{n}_{\mathbf{r}}\right)}{\mathbf{N}\left(\mathbf{n}_{\mathbf{r}}\right)}$

24. Calculate forwarding probability:

25. $\operatorname{Pfw}(\mathbf{n r})=\operatorname{Cmetric}(\mathbf{n r}) \times \operatorname{Acov}-\operatorname{ratio}(\mathbf{n r})$

26. If random $(0,1) \leq$

$\operatorname{Pfw}(\mathbf{n r})$

27. Rebroadcast msg

28. Else

29. Discard msg

30. End if 


\section{Performance Analysis}

\subsection{Simulation Scenarios and Parameters}

We have made an effort to examine and assess the effectiveness of our proposed NCKPB model for an approach to FL and FP. All routing schemes are investigated under three main operating scenarios of the MANET network, such as speed, density and load. Mobile nodes are uniformly dispersed across 1000 x 1000 metres of geographical region with random way point mobility model (RWP) [8]. For each network node the probability of retransmission varies from 0 to 1 . The simulation period for all three scenarios is set at 900 seconds. First, we performed detailed simulation to study the density impacts, where the amount of nodes ranging from 75 to 200 nodes. The second scenario analysis explores the effect of high speed on three separate routing protocols. 150 numbers of mobile hosts are distributed uniformly to study the influence on the mobility. The mobility of the node scales from 5 to $30 \mathrm{~m} / \mathrm{sec}$ with zero pauses. The third aspect of network analysis discusses the outcomes of the traffic loads provided. 125 node numbers are distributed uniformly to evaluate the effect of load differences from 5 to 25 CBR connections. The node speed chosen is $10 \mathrm{~m} / \mathrm{sec}$ with zero pause time for both traffic load and node density scenarios. Maximum of 10 CBR links are arbitrarily chosen for traffic load.

Table 1. Simulation parameters

\begin{tabular}{|l|l|}
\hline Simulation Parameter & Value \\
\hline Simulator & NS2 \\
\hline MAC protocol & 802.11 \\
\hline Topology size & $1000 \mathrm{~m}$ x $1000 \mathrm{~m}$ \\
\hline Radio propagation model & Two-ray ground \\
\hline Node's transmission radius & $250 \mathrm{~m}$ \\
\hline Bandwidth & $2 \mathrm{mbps}$ \\
\hline Interface queue length & 50 packets \\
\hline Mobility model & Random way point \\
\hline Traffic type & Constant Bit Rate $(\mathrm{CBR})$ \\
\hline Packet length & 512 bytes \\
\hline Packet transmission rate & 4 packets/second \\
\hline Number of Nodes & $75,100,125,150,175,200$ \\
\hline Maximum node's speed & $5,10,15,20,25,30 \mathrm{~m} / \mathrm{s}$ \\
\hline Number of traffic flows & $5,10,15,20,25$ \\
\hline Pause time & 0 second $($ Continuous mobility) \\
\hline Simulation time & 900 seconds \\
\hline
\end{tabular}

\subsection{Performance Metrics}

We have assessed three different broadcasting schemes using following performance metrics: 


\subsubsection{Throughput}

Throughput measures the amount of successful data packets that are transmitted in a given amount of time over a communication channel.

$$
\text { Throughput }=\frac{\sum_{1}^{\mathrm{n}} \text { Data packets received } \mathrm{x} \text { Packet size }}{\text { Total simulation time }}
$$

\subsubsection{End-to-End delay (ENE)}

Average delay is the amount of time the data packet spends to reach at the destination.

\subsubsection{RREQ overhead}

$$
\mathrm{ENE}=\frac{\sum_{1}^{\mathrm{n}}(\text { Sent time-Received time })}{\sum_{1}^{\mathrm{r}} \text { Data packets received }}
$$

It defines the amount of route request (RREQ) packets propagated over the simulation period.

$$
\text { RREQ Overhead }=\sum_{1}^{\mathrm{n}} \mathrm{RREQ} \text { packets transmitted }
$$

\subsubsection{MAC collision}

This metric determines the number of data packets (CBR) which are dropped in collisions per second over the MAC layer.

$$
\text { MAC Collision }=\sum_{1}^{\mathrm{n}} \text { Data packets dropped at MAC }
$$

\subsubsection{Reachability (RE)}

It is the fraction of the nodes received the broadcasted RREQ packets over the total amount of nodes in the network.

$$
\mathrm{RE}=\frac{\sum_{1}^{\mathrm{x}} \text { Nodes received the } \mathrm{RREQ}}{\text { Total network nodes }}
$$

\subsubsection{Saved Rebroadcast (SRB)}

This metric calculates the ratio of RREQ packets received minus transmitted over the total amount of RREQ packets received in the network.

$$
\mathrm{SRB}=\frac{\sum_{1}^{\mathrm{r}} \text { Nodes received }-\sum_{1}^{\mathrm{t}} \text { Nodes transmitted }}{\sum_{1}^{\mathrm{r}} \text { Nodes received }}
$$




\subsection{Performance Results}

\subsubsection{Effects of Network Density}

\subsubsection{Routing Overhead}

The effect of node densities on RREQ packet retransmission is plotted in Figure 1 for three protocols. During the simulation study, the redundancy overhead in the network reflects the amount of RREQ messages produced and propagated. Performance outcome of overhead routing shows that NCKPB surpasses the other two schemes, while FP outperforms FL. Figure 1 shows that as the network density grows from 75 to 200 numbers of nodes, all the protocols severely suffers with increased number of RREQ overheads. Overall analysis clearly indicates that the volume of RREQ packets transmitted across the network for NCKPB is lowered by $35 \%$ and $56 \%$ contrasts to FP and FL.

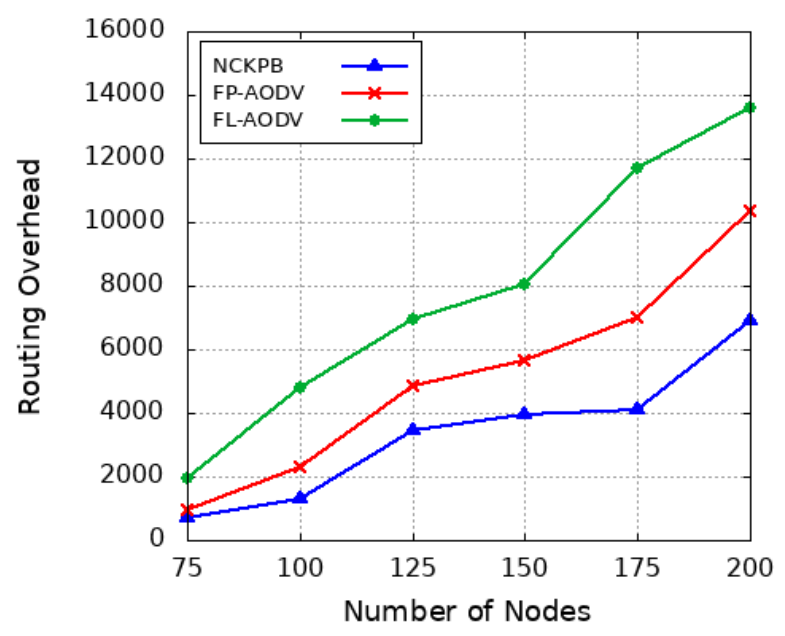

Figure 1. Routing Overhead vs. Number of Nodes

\subsubsection{Collision}

The effect of the MAC collision on all three routing schemes is seen in Figure 2. With the increase of node density from 75 to 150 numbers of nodes, the number of collisions increases significantly. It is obvious because the average percentage of RREQ overhead retransmissions substantially grows higher with each of the routing protocol. Ultimately, the individual node's bandwidth gets impaired and ultimately raises the risk of packet loss and drop. The NCKPB protocol demonstrates its superiority over the other two methods of routing. The simulation findings specifically indicate that the overall collision rate for NCKPB is reduced by $33 \%$ and 53\% compared to FP and FL. 
International Journal of Computer Networks \& Communications (IJCNC) Vol.13, No.2, March 2021

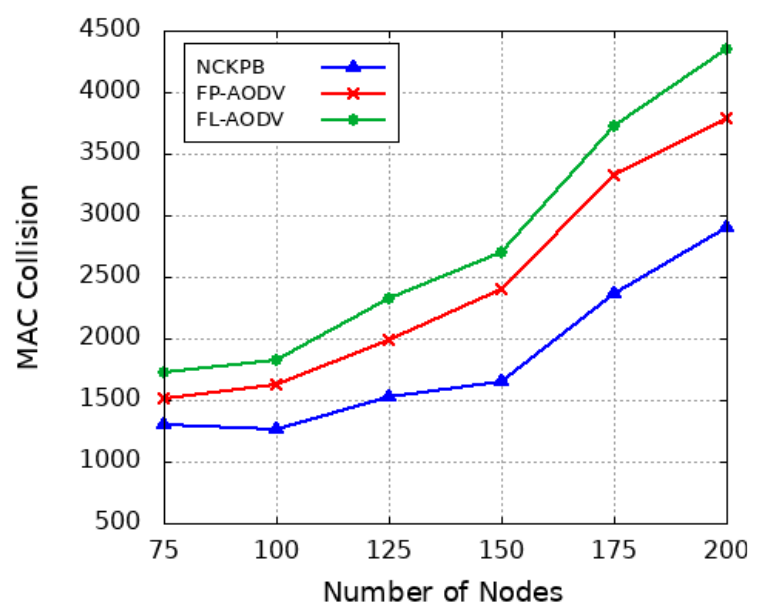

Figure 2. MAC Collision vs. Number of Nodes

\subsubsection{End to End Delay}

Figure 3 reflects the delay performance of three distinct routing schemes with increased network density. It is observed that average delay gets increased with the increased network density. The reason for the increased delay is attributed primarily to excessive collisions, channel contention and the amount of retransmissions in the MAC layer due to unwanted drop. This raises the delay in reaching the destination of the CBR packets. Enhanced routing efficiency with lower transmission of RREQ packets increases delay performance. The simulation results indicate that compared to the other two variants, the volume of broadcast retransmissions is considerably smaller with the proposed NCKPB model and has proved to be a better routing mechanism. This effectively ensures the efficiency of the NCKPB algorithm by decreasing the delay to $25 \%$ and $35 \%$ contrast to FP and FL.

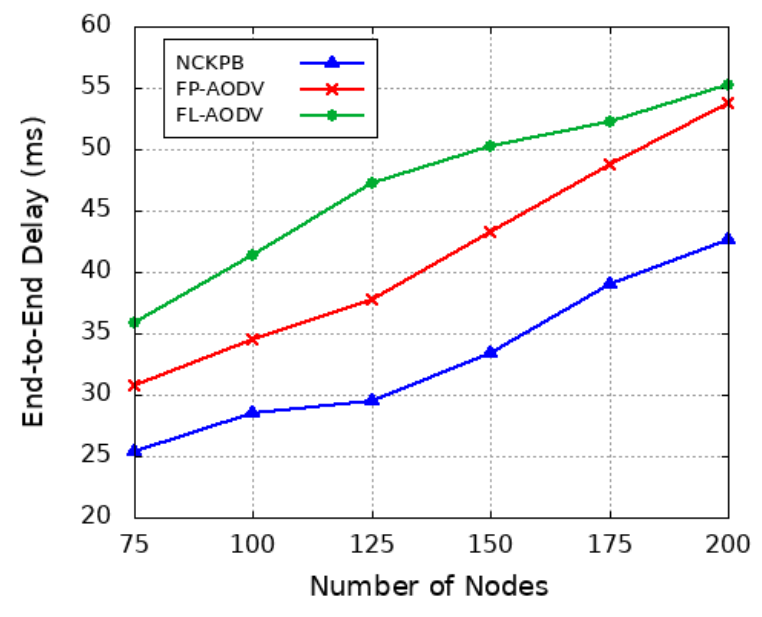

Figure 3. End-to-End Delay vs. Number of Nodes

\subsubsection{Throughput}

The findings in Figure 4 depict the average throughput performance among all the routing protocols with node density. Results clearly reveal that the average throughput of the network decreases with increased network density. It is obvious, because higher number of redundancy control overheads contributes to excessive network congestion. Probability of packets collisions and contention of the 
channel severely affects throughput performance of all the protocols. The average range of throughput performance lies between $17 \mathrm{kbps}$ to $16.78 \mathrm{kbps}$ for NCKPB, $16.95 \mathrm{kbps}$ to $17.75 \mathrm{kbps}$ for FP and $16.91 \mathrm{kbps}$ to $16.7 \mathrm{kbps}$ for FL with the differences in network density. The proposed model NCKPB shows its superiority over throughput performance due its combined advantage of neighbour knowledge and controlled transmission of broadcasting. Outcome results specifically reveal that the total average throughput performance achieved by NCKPB, FP and FL is 17.03 kbps, $16.83 \mathrm{kbps}$ and $16.78 \mathrm{kbps}$ respectively.

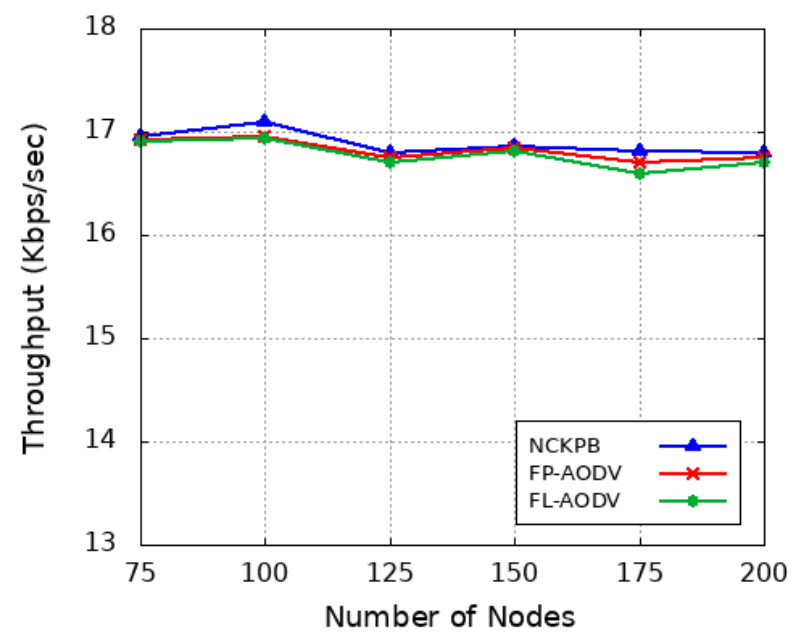

Figure 4. Throughput vs. Number of Nodes

\subsubsection{Reachability (RE)}

Figure 5 reveals the influence of various node densities on the simulation's reachability. From the results of the simulation it is found that comparison to FP and FL, NCKPB performs well without compromising reachability. Simulation results explicitly indicate that the ratio of nodes obtaining the RREQ message across the network is growing steadily with node density. NCKPB, FP and FL have an average reachability performance of $97.6 \%, 95.4 \%$, and $99.6 \%$ respectively. It is obvious for the proposed NCKPB that the retransmission probabilities are dynamically regulated by each network node resulting in improved results compared to FP.

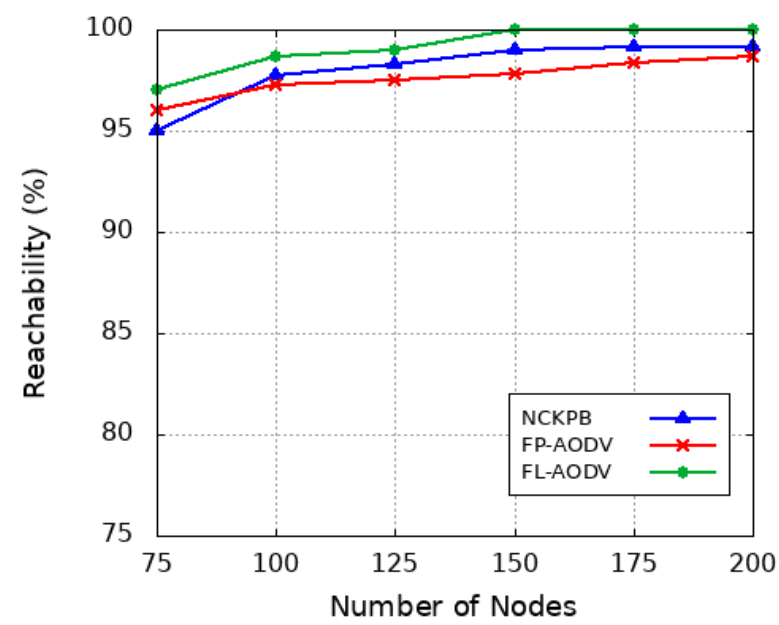

Figure 5. Packet Reachability vs. Number of Nodes 


\subsubsection{Saved Rebroadcast (SRB)}

Figure 6 demonstrates the FP and FL saving rebroadcast performance over proposed NCKPB routing algorithm. This saved rebroadcast metric measures the proportion of duplicate messages that can be saved from the total network RREQ generated. The higher the amount of rebroadcast saved, the better the protocol performance. However, sending very few rebroadcast packets induces poor reachability, which eventually contributes to a lack of protocol functionality. The number of broadcast transmissions substantially grows with increased density. NCKPB's saved re-broadcast performance improves linearly whereas no improvement is found either in blind flooding or fixed probabilistic scheme. Because of NCKPB's dynamic neighbouring information probability broadcast scheme, it tends to save redundancy broadcasting at low density by around $40 \%$ and gradually saves up to $60 \%$ in higher node density networks. The total percentage of NCKPB's saved rebroadcast packets is approximately more than 52\% of the total RREQ generated and transmitted over the network.

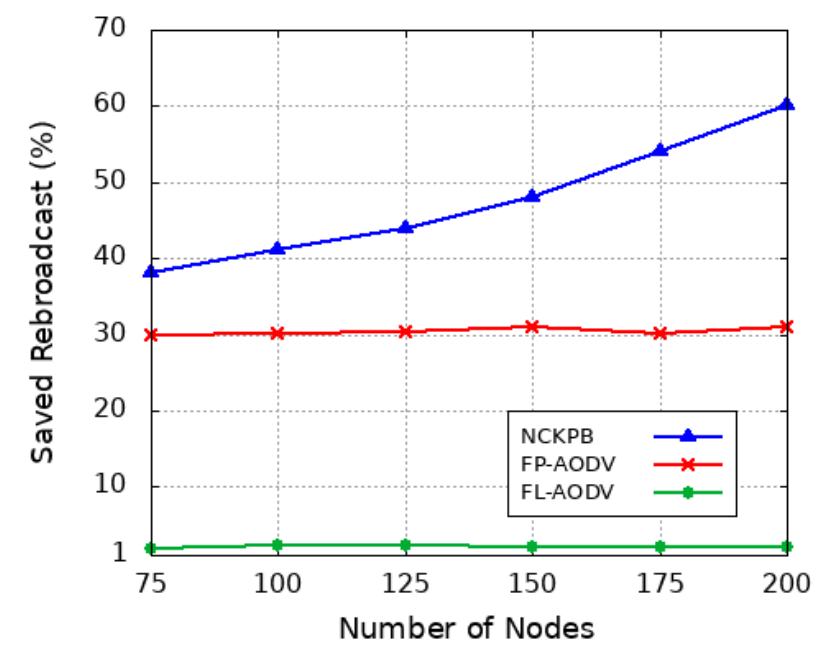

Figure 6. Saved Rebroadcast vs. Number of Nodes

\subsubsection{Effects of Node Mobility}

\subsubsection{Routing Overhead}

Figure 7 exhibits the performance measure of RREQ overheads on three separate routing variants against node mobility. Overhead routing transmissions gradually increases with every increase in node speed ( 5 to $20 \mathrm{~m} / \mathrm{s}$ ). In contrast, an increase in the mobility beyond $20 \mathrm{~m} / \mathrm{s}$ (i.e. 25 and $30 \mathrm{~m} / \mathrm{s}$ ) is reported to have a positive effect on all the routing schemes. Because higher node speed significantly reduces the path length between the nodes. The connectivity becomes stronger and the probability of link failures and its repair gradually decreases. It is observed that our proposed model NCKPB works better in high speed network. The experimental findings specifically demonstrate that the average routing overhead of NCKPB is greatly decreased by $30 \%$ and $42 \%$ relative to FP and FL schemes. 
International Journal of Computer Networks \& Communications (IJCNC) Vol.13, No.2, March 2021

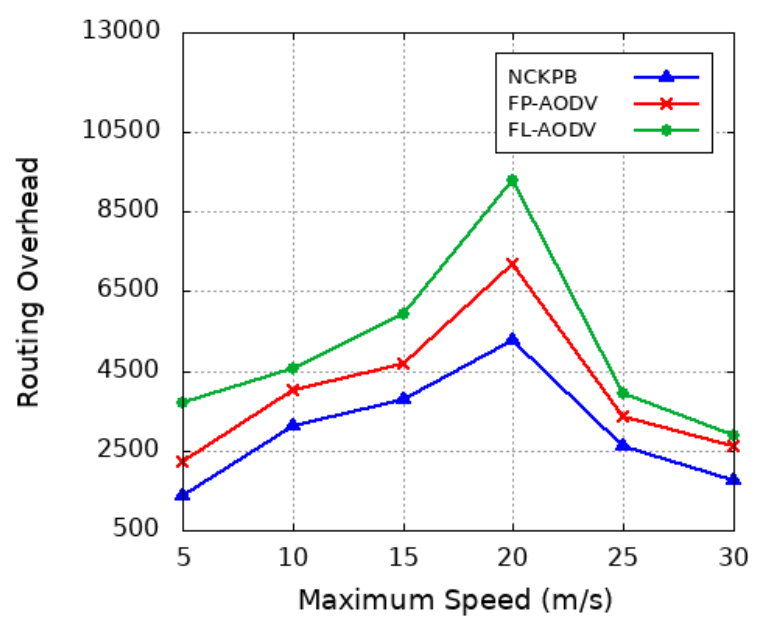

Figure 7. Routing overhead vs. Maximum Speed for 150 nodes

\subsubsection{Collision}

The simulation result in Figure 8 demonstrates the node mobility impact of routing protocols on packet collisions. Findings clearly represents that the rate of collisions gradually rises due to rapid increase in overhead transmissions. With low to moderate node mobility ( 5 to $20 \mathrm{~m} / \mathrm{s}$ ), the path length gets increased between source to destination pairs. And eventually, the network struggles from factors such as severe interference and strong congestion. However, higher node mobility causes a favourable impact on protocol routing. The redundancy broadcast retransmissions are greatly reduced due to the dynamic control mechanism of the NCKPB, thereby increasing the performance of the protocols for all the metrics considered. Our proposed NCKPB eliminates packet collisions by more than $29 \%$ and $40 \%$ relative to FP and FL.

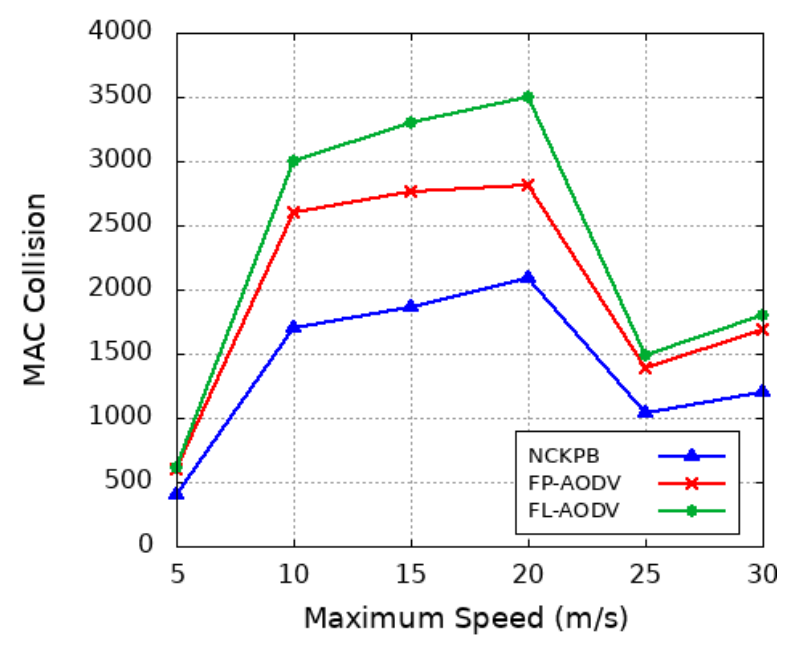

Figure 8. MAC Collision vs. Maximum Speed for 150 nodes

\subsubsection{End to End Delay}

The simulation findings in Figure 9 display the delay performance of NCKPB against other two variants with respect to speed. It is clearly evident that the average delay parameter rises with rise in node mobility. The delay increased by $22 \%$ for NCKPB, $35 \%$ for FP and $45 \%$ for FL with every increase of speed $(5-20 \mathrm{~m} / \mathrm{s})$. The reason of such delays is attributed to a variety of factors, such as 
delays during path compilation, packet queuing times, and propagation and transmission delays to the MAC layer. Higher collision and packet loss, all these factors imply larger delays. With higher node speed (i.e. 25 and $30 \mathrm{~m} / \mathrm{s}$ ), the node moves faster towards the outer node, thereby reducing the length of the route between the intermediate nodes. A secure network is less susceptible to connectivity loss and thus creates less control overheads. As a result, network queue data packets do not have a long wait period to reach the target. The proposed NCKPB model reveals its superiority in reducing delay performance by approximately more than $30 \%$ and $42 \%$, respectively compared to FP and FL.

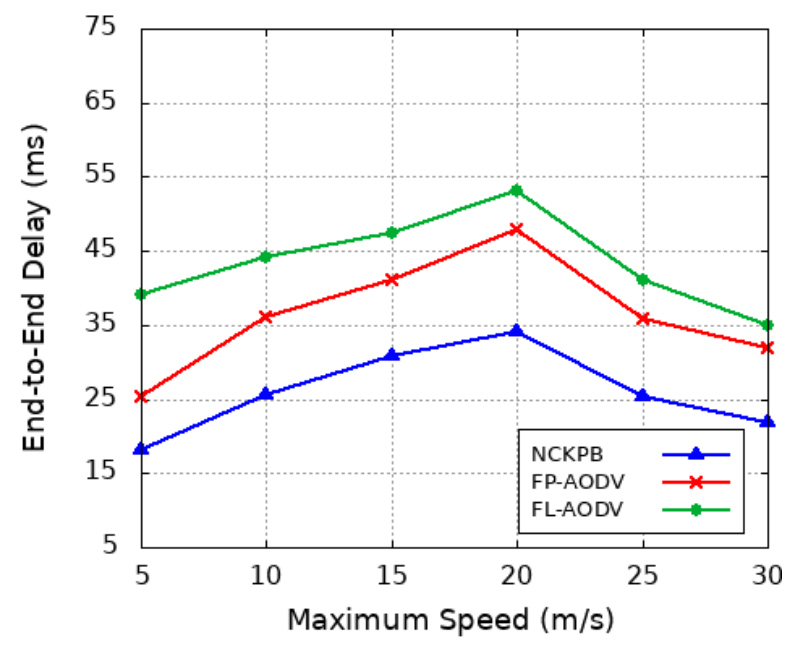

Figure 9. End-to-End Delay vs. Maximum Speed for 150 nodes

\subsubsection{Throughput}

The simulation results illustrated in Figure 10 indicate throughput over mobility for three routing protocols. Outcome indicates that the performance of the throughput over all protocols is slightly decreased from 5 to $20 \mathrm{~m} / \mathrm{s}$ for each increase in speed. Huge amount of overhead controls to create a precise routing table (RT) that essentially affects the bandwidth and delay of the individual nodes. At higher node mobility $(25 \mathrm{~m} / \mathrm{s})$, all protocols function well, enabling greater network connectivity. NCKPB, FP and FL schemes have an optimum throughput performance of 13.05 kbps, $12.96 \mathrm{kbps}$ and $12.91 \mathrm{kbps}$, respectively, at a speed of $25 \mathrm{~m} / \mathrm{s}$. The efficiency of the throughput really depends on the delay and channel bandwidth. The findings obtained explicitly indicate that NCKPB is dominant in terms of the throughput rate compared to others. The average throughput rate over simulation for NCKPB is $12.93 \mathrm{kbps}$, for FP $12.9 \mathrm{kbps}$ and for FL $12.85 \mathrm{kbps}$. 
International Journal of Computer Networks \& Communications (IJCNC) Vol.13, No.2, March 2021

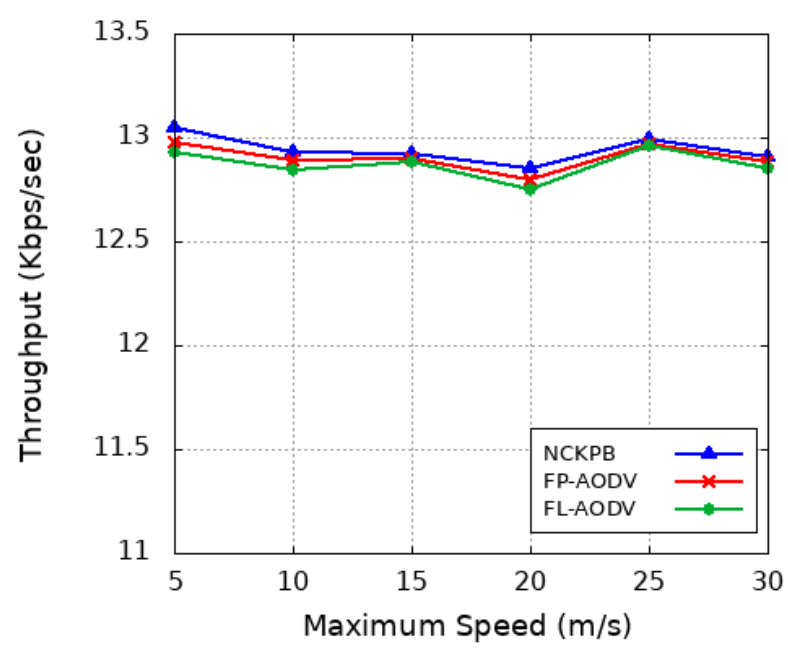

Figure 10. Throughput vs. Maximum Speed for 150 nodes

\subsubsection{Reachability (RE)}

Figure 11 displays the findings with respect to reachability for all routing protocols against node mobility. Specifically, the results indicate that performance in terms of reachability is marginally compromised, irrespective of routing schemes. The flood strategy ensures all mobile hosts receive packets sent at the risk of enhanced traffic generated by redundant RREQs. Conversely, redundant messages even result in higher collisions, which ultimately cause immense message drops, thereby directly impacting reachability. At a lower speed network, each node has a higher level of neighbouring nodes. The network is more influenced by overhead control, collisions and additional delays. The outcome over the entire simulation shows that the NCKPB reachability is $96 \%$, FP is $97.8 \%$ and FL is $99 \%$ respectively.

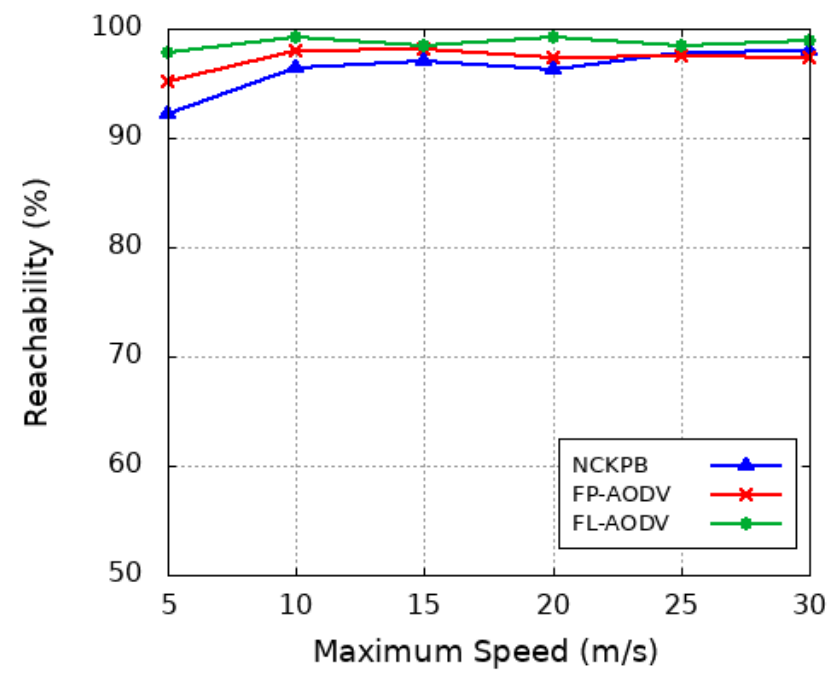

Figure 11. Packet Reachability vs. Maximum Speed for 150 nodes 


\subsubsection{Saved Rebroadcast (SRB)}

Figure 12 indicates the average rebroadcasting performance of NCKPB compared to FP and FL with varying speeds. The lower speed network is more liable to stagnate and the amount of adjacent nodes per node is greater. The generation and propagation of overhead routing is higher to ensure the efficient data communications to the destination. The NCKPB model reached a ratio of more than approximately $50 \%$ SRB from 5 to $20 \mathrm{~m} / \mathrm{s}$ of node mobility. In contrast, faster speeds (i.e. 25 $30 \mathrm{~m} / \mathrm{s}$ ) have a predictably favourable impact on the efficiency of the proposed model. Greater speed ensures substantially lower overhead routing when the length of the route from source to destination is shortened. Higher speed network experiences less overhearing between participating nodes that minimises congestion and improves efficiency. The overall saving of rebroadcast for NCKPB is more than 52\%, whereas no improvement is found either in FL or FP scheme.

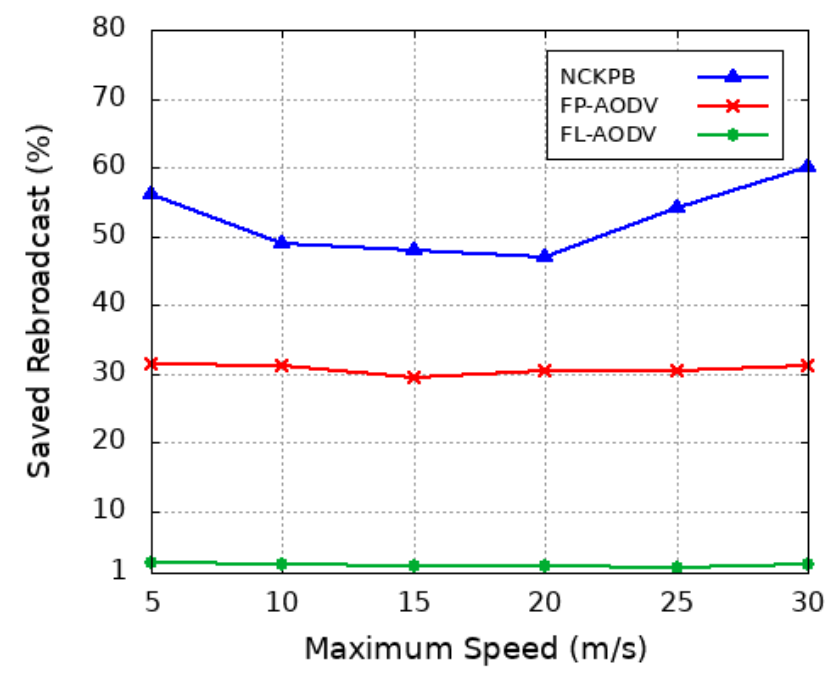

Figure 12. Saved Rebroadcast vs. Maximum Speed for 150 nodes

\subsubsection{Effects of Traffic Load}

\subsubsection{Routing Overhead}

Findings represented in Figure 13 presents the RREQ overhead incurred by NCKPB, FP and FL for 5 to 25 flows of CBR connections. Experimental outcomes on traffic load reveal that NCKPB has distinct performance gain compared to other two variants. More fresh paths are required when the number of source-destination pairs have been increased. It triggers a proportionate number of new path discoveries to carry the CBR packets to their destination. As a response, enormous amount of RREQ packets are produced that have a negative impact on all performance metrics. In overall, the total volume of redundant RREQ retransmissions of NCKPB decreased by around $25 \%$ and $50 \%$ relative to FP and FL for variable traffic load. 
International Journal of Computer Networks \& Communications (IJCNC) Vol.13, No.2, March 2021

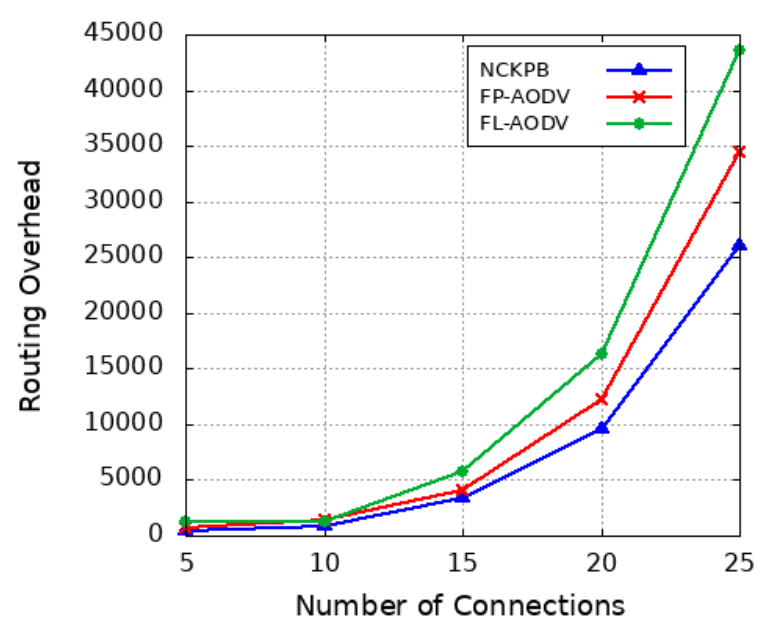

Figure 13. Routing Overhead vs. Traffic Load for 125 nodes

\subsubsection{Collision}

In Figure 14, the effect of traffic load on packet collisions is demonstrated. Subsequent results of simulations revealed that for all different loads from 5 to 25 (Max connections), NCKPB followed by FP performs better than FL. Compared to other two variants, NCKPB demonstrates superiority over collision rate among other two protocols. This is attributed to the successful framework of the proposed model with a fair retransmission delay that dynamically governs the retransmission probability of nodes. The simulation result clearly illustrates that with all connection pairs, NCKPB beats collision reduction by $26 \%$ and $33 \%$ compared to FP and FL.

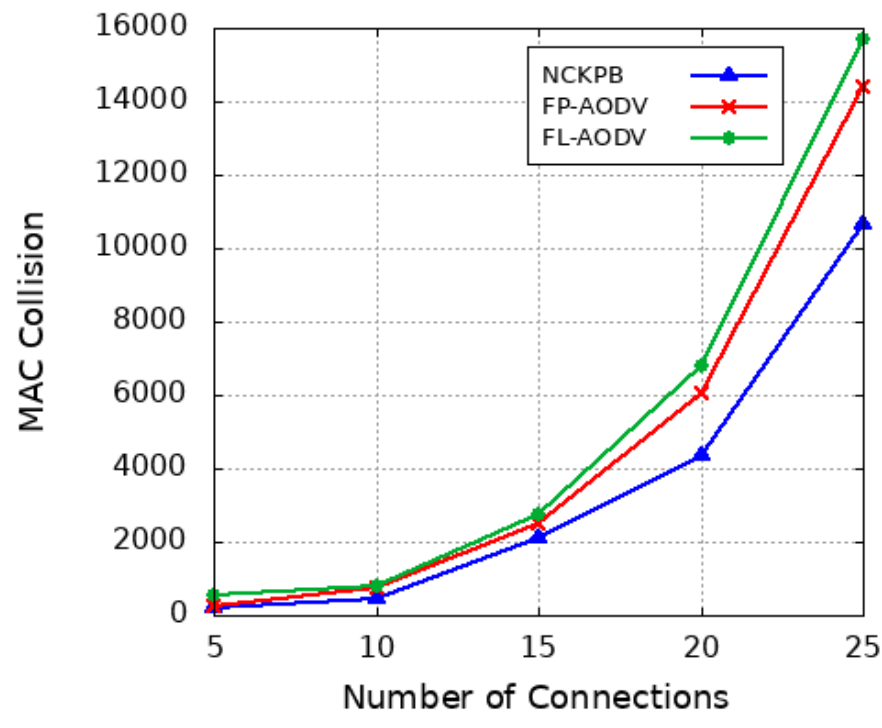

Figure 14. MAC Collision vs. Traffic Load for 125 nodes

\subsubsection{End to End Delay}

NCKPB's average latency performance, contrasting with FP and FL with varying traffic loads has been illustrated in Figure 15. With the increased number of maximum connections, the delay measures have been significantly impacted. It is clear, as the overhead redundancy is significantly enhanced with traffic load variations. These redundant RREQ packets overload the network queue, 
collisions and channel contentions. The network still struggles from longer delays from source to destination. Such overhead redundancy involves the transfer of packets suffering from elevated delay for extra time in the network queue. The NCKPB incurred the lowest overall end to end delay of $30 \%$ and $44 \%$ respectively relative to FP and FL.

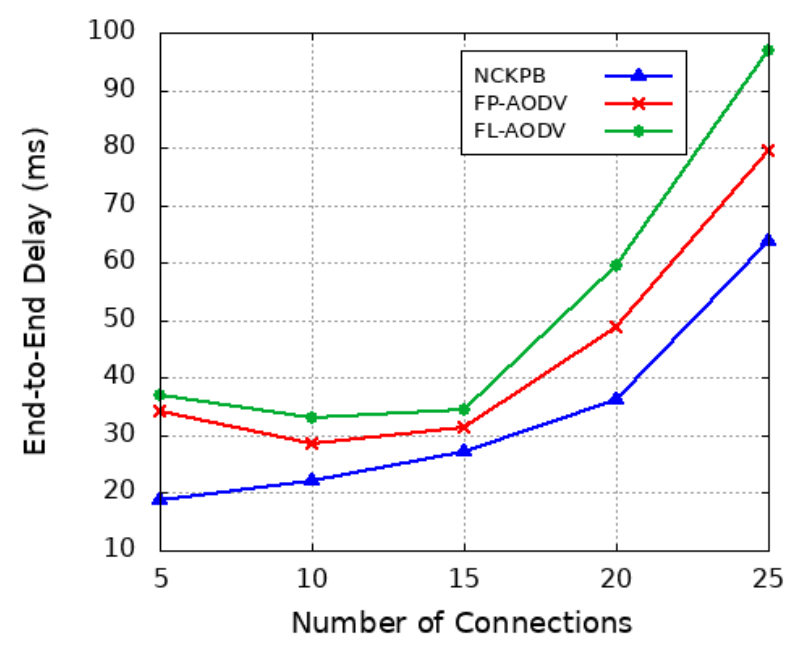

Figure 15. End-to-End Delay vs. Traffic Load for 125 nodes

\subsubsection{Throughput}

The findings in Figure 16 indicate that throughput performance is fairly comparable for all the routing protocols. The increase in average throughput of all the distinct routing variants increases with increase in connection pairs (5 to 25 max connections). The throughput performance of NCKPB dominates over other two variants. NCKPB reaches optimum throughput of $29.5 \mathrm{kbps}$, $28.27 \mathrm{kbps}$ for FP and $28.72 \mathrm{kbps}$ for FL, respectively at the traffic load of 25 CBR connections. The average network throughput rating of NCKPB is $18.5 \mathrm{kbps}, 18.35 \mathrm{kbps}$ for FP and $18.21 \mathrm{kbps}$ for FL at low and high traffic loads (i.e. 5-25 source-destination pairs).

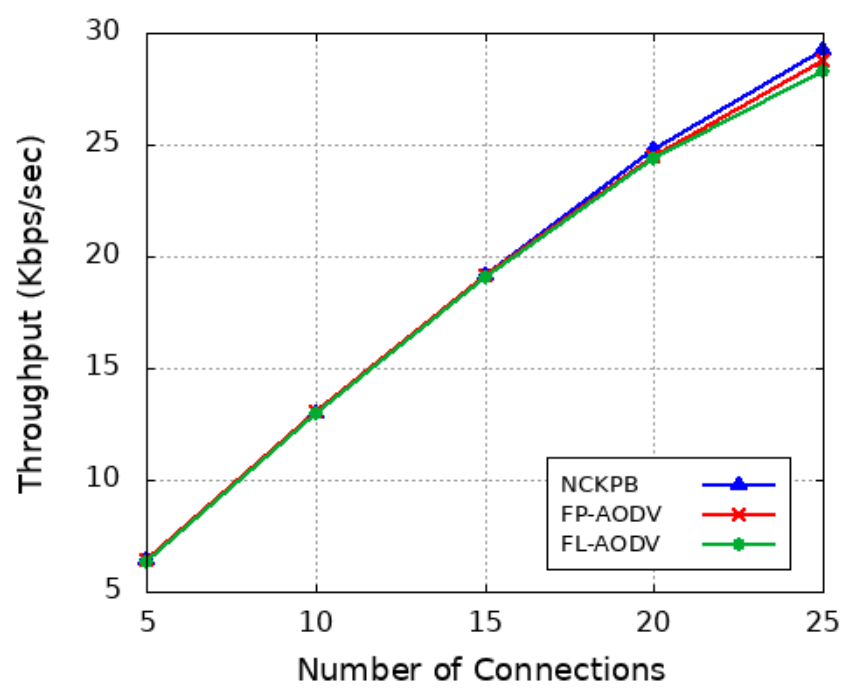

Figure 16. Throughput vs. Traffic Load for 125 nodes 


\subsubsection{Reachability (RE)}

Figure 17 illustrates the effect on the reachability of the simulation of different traffic loads. Flooding system ensures that any node in the network receives packets at the cost of redundant overheads to reach optimum connectivity. However, the finding indicates that the reachability performance of simple flooding is also compromised by distinct traffic flows. In contrast, our latest NCKPB protocol, on the other side, is a good way to retain broad reach. The NCKPB's reachability improves with more nodes. The average percentage of reachability attained by NCKPB is $94.5 \%$, $\mathrm{FP}$ is $96 \%$ and $\mathrm{FL}$ is $97 \%$.

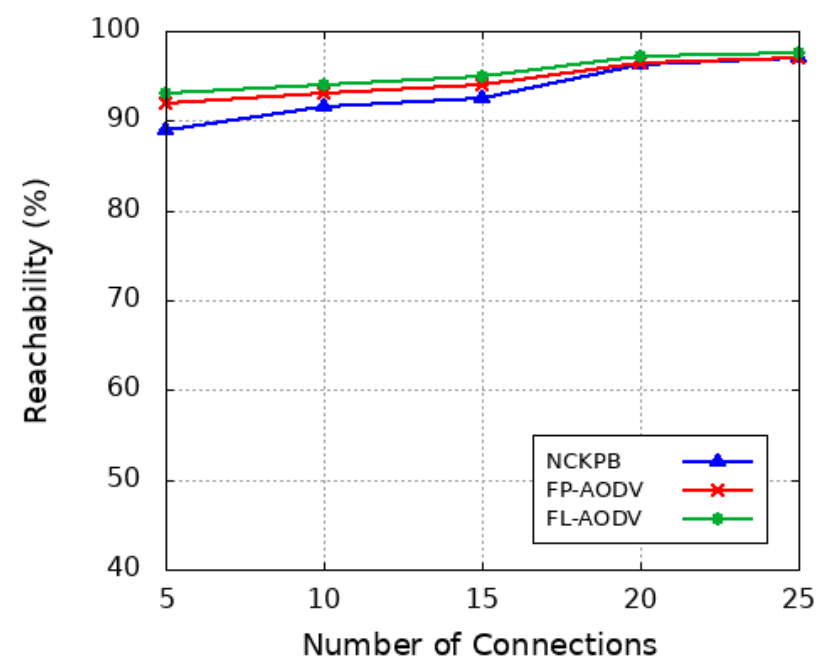

Figure 17. Packet Reachability vs. Traffic Load for 125 nodes

\subsubsection{Saved Rebroadcast (SRB)}

Figure 18 demonstrates the effect of traffic load variations on saved rebroadcasts for all variants. Traffic loads vary from 5 to 25 CBR source-destination pairs. With increased source-destination pairs, each coverage region has a higher number of retransmission candidates. Proportionate volumes of RREQ packet transfers are required for new route discoveries. Simulation findings clearly indicate that the performance of NCKPB with respect to SRB metrics continues to improve with each increase in traffic load, with the exception of a marginal loss of performance at $20 \mathrm{CBR}$ connections. However, better performance improvements are found when the traffic flow is heavier (25 CBR connections). Contrast to other two variants, our proposed scheme NCKPB has its dominance and the average ratio of saved rebroadcasts is more than $45 \%$ over the generated and retransmitted overhead RREQ in the network. 
International Journal of Computer Networks \& Communications (IJCNC) Vol.13, No.2, March 2021

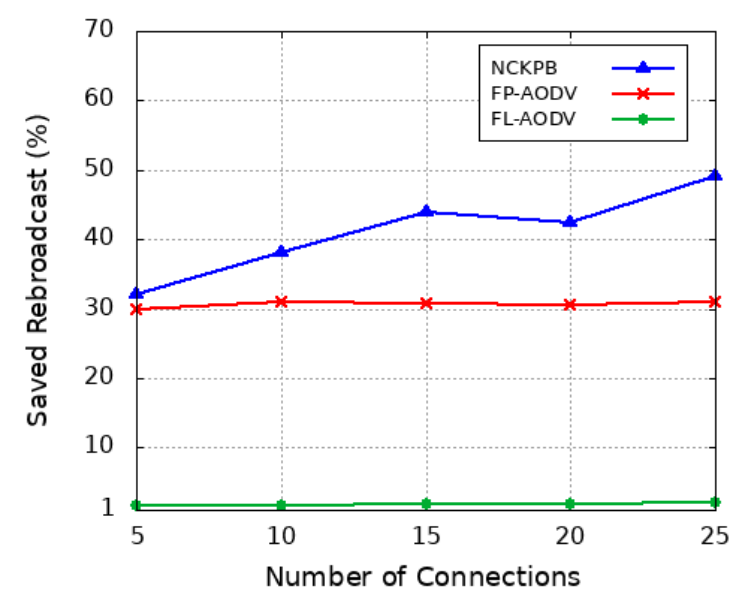

Figure 18. Saved rebroadcast vs. Traffic Load for 125 nodes

\section{Conclusions}

Extensive simulations using NS-2 are performed to analyze the three routing schemes NCKPB, FP and FL in various network scenarios such as network densities, traffic loads and mobility. Our main goal of this research analysis is to develop a model that blends the advantages of knowledge of neighbourhood coverage with the probabilistic method. The proposed scheme bypasses the conventional way of route discovery process usually found in AODV. Excessive redundant RREQ retransmissions and collisions are significantly reduced in broadcasting using the following procedures: estimating a reasonable rebroadcast delay for a node, acquisition of neighbouring information to determine additional coverage area, a connectivity function to regulate the probability of retransmissions. The experimental findings show that in all network situations, NCKPB proves its dominance over two other variants to minimize the diffusion storm problems. The simulation outcome demonstrates that our well-designed model NCKPB greatly reduce the RREQ overhead with tremendous enhancement in terms of QoS performance metrics such as MAC collision, throughput, delay, reachability and saved rebroadcast. In the further scope of our research study, we intend to implement a more robust adaptive probabilistic scheme, taking into account an optimal path selection using cost function of various routing metrics that guarantees a committed level of QoS.

\section{CONFLICTS OF INTEREST}

The authors declare no conflict of interest.

\section{REFERENCES}

[1] Murthy, C. S. R., \& Manoj, B. S. (2004). Ad hoc wireless networks: Architectures and protocols, portable documents. Pearson education.

[2] Toh, C. K. (2001). Ad hoc mobile wireless networks: protocols and systems. Pearson Education.

[3] Perkins, C., Belding-Royer, E., \& Das, S. (2003). RFC3561: Ad hoc on-demand distance vector (AODV) routing..

[4] Johnson, D., Hu, Y. C., \& Maltz, D. (2007). The dynamic source routing protocol (DSR) for mobile ad hoc networks for IPv4 (Vol. 260). RFC 4728.

[5] Perkins, C. E., \& Bhagwat, P. (1994). Highly dynamic destination-sequenced distance-vector routing (DSDV) for mobile computers. ACM SIGCOMM computer communication review, 24(4), 234-244.

[6] Haas, Z. J., Pearlman, M. R., \& Samar, P. (1999). Zone routing protocol for ad-hoc networks. Internet Draft, draft-ietf-manet-zrp-02. txt, work in progress. 
[7] Das, S. R., Perkins, C. E., \& Royer, E. M. (2000, March). Performance comparison of two on- demand routing protocols for ad hoc networks. In Proceedings IEEE INFOCOM 2000. Conference on Computer Communications. Nineteenth Annual Joint Conference of the IEEE Computer and Communications Societies (Cat. No. 00CH37064) (Vol. 1, pp. 3-12). IEEE.

[8] Camp, T., Boleng, J., \& Davies, V. (2002). A survey of mobility models for ad hoc network research. Wireless communications and mobile computing, 2(5), 483-502.

[9] The NS Manual (Formerly NS Notes and Documentation) (2011) The VINT Project: A Collaboration between Researchers at UC Berkeley, LBL, USC/ISI, Xerox PARC. 5 November.

[10] Tseng, Y. C., Ni, S. Y., Chen, Y. S., \& Sheu, J. P. (2002). The broadcast storm problem in a mobile ad hoc network. Wireless networks, 8(2-3), 153-167.

[11] Tseng, Y. C., Ni, S. Y., \& Shih, E. Y. (2003). Adaptive approaches to relieving broadcast storms in a wireless multihop mobile ad hoc network. IEEE transactions on computers, 52(5), 545-557.

[12] Lim, H., \& Kim, C. (2001). Flooding in wireless ad hoc networks. Computer Communications, 24(3-4), 353-363.

[13] Williams, B., \& Camp, T. (2002, June). Comparison of broadcasting techniques for mobile ad hoc networks. In Proceedings of the 3rd ACM international symposium on Mobile ad hoc networking \& computing (pp. 194-205).

[14] Sasson, Y., Cavin, D., \& Schiper, A. (2003, March). Probabilistic broadcast for flooding in wireless mobile ad hoc networks. In 2003 IEEE Wireless Communications and Networking, 2003. WCNC 2003. (Vol. 2, pp. 1124-1130). IEEE.

[15] Reina, D. G., Toral, S. L., Johnson, P., \& Barrero, F. (2015). A survey on probabilistic broadcast schemes for wireless ad hoc networks. Ad Hoc Networks, 25, 263-292.

[16] Zhang, Q., \& Agrawal, D. P. (2005). Dynamic probabilistic broadcasting in MANETs. Journal of parallel and Distributed Computing, 65(2), 220-233.

[17] Bani-Yassein, M., Ould-Khaoua, M., Mackenzie, L. M., \& Papanastasiou, S. (2006). Performance analysis of adjusted probabilistic broadcasting in mobile ad hoc networks. International journal of wireless information networks, 13(2), 127-140.

[18] Hanashi, A. M., Siddique, A., Awan, I., \& Woodward, M. (2009). Performance evaluation of dynamic probabilistic broadcasting for flooding in mobile ad hoc networks. Simulation Modelling Practice and Theory, 17(2), 364-375.

[19] Abdulai, J. D., Ould-Khaoua, M., Mackenzie, L. M., \& Mohammed, A. (2008, June). Neighbour coverage: A dynamic probabilistic route discovery for mobile ad hoc networks. In 2008 International Symposium on Performance Evaluation of Computer and Telecommunication Systems (pp. 165-172). IEEE.

[20] Yassein, M. B., Khaoua, M. O., Mackenzie, L. M., Papanastasiou, S., \& Jamal, A. (2006, October). Improving route discovery in on-demand routing protocols using local topology information in MANETs. In Proceedings of the ACM international workshop on Performance monitoring, measurement, and evaluation of heterogeneous wireless and wired networks (pp. 95-99).

[21] Lim, H., \& Kim, C. (2000, August). Multicast tree construction and flooding in wireless ad hoc networks. In Proceedings of the 3rd ACM international workshop on Modeling, analysis and simulation of wireless and mobile systems (pp. 61-68).

[22] Peng, W., \& Lu, X. C. (2000, August). On the reduction of broadcast redundancy in mobile ad hoc networks. In 2000 First Annual Workshop on Mobile and Ad Hoc Networking and Computing. MobiHOC (Cat. No. 00EX444) (pp. 129-130). IEEE.

[23] Kim, J. S., Zhang, Q., \& Agrawal, D. P. (2004, November). Probabilistic broadcasting based on coverage area and neighbor confirmation in mobile ad hoc networks. In IEEE Global Telecommunications Conference Workshops, 2004. GlobeCom Workshops 2004. (pp. 96-101). IEEE.

[24] Zhang, X. M., Wang, E. B., Xia, J. J., \& Sung, D. K. (2012). A neighbor coverage-based probabilistic rebroadcast for reducing routing overhead in mobile ad hoc networks. IEEE transactions on mobile computing, 12(3), 424-433.

[25] Ejmaa, A. M. E., Subramaniam, S., Zukarnain, Z. A., \& Hanapi, Z. M. (2016). Neighbor-based dynamic connectivity factor routing protocol for mobile ad hoc network. IEEE Access, 4, 8053-8064.

[26] Rab, R., Sagar, S. A. D., Sakib, N., Haque, A., Islam, M., \& Rahman, A. (2017). Improved self- pruning for broadcasting in ad hoc wireless networks. Wireless Sensor Network, 9(2), 73-86.

[27] Lu, D., \& Dong, S. (2017). A neighbor knowledge and velocity-based broadcast scheme for wireless ad hoc networks. International Journal of Distributed Sensor Networks, 13(11), 1550147717743699.

[28] Tizvar, R., \& Abbaspour, M. (2020). A density-aware probabilistic interest forwarding method for contentcentric vehicular networks. Vehicular Communications, 23, 100216.

[29] Xu, H., Si, H., Zhang, H., Zhang, L., Leng, Y., Wang, J., \& Li, D. (2020). Trust-Based Probabilistic Broadcast Scheme for Mobile Ad Hoc Networks. IEEE Access, 8, 21380-21392. 
International Journal of Computer Networks \& Communications (IJCNC) Vol.13, No.2, March 2021

[30] Pallai, G. K., Meenakshi, S., Rath, A. K., \& Majhi, B. (2015). Comparative Study of On- Demand and Table-Driven Routing Protocols in MANET. In Computational Intelligence in Data Mining-Volume 2 (pp. 369-379). Springer, New Delhi.

[31] Pallai, G. K., Meenakshi, S., Rath, A. K., \& Majhi, B. (2014). A Quantitative Analysis and Behavioral Study of Routing Protocols in MANET.

\section{AUTHOR}

Prof. Gyanendra Kumar Pallai is an alumni of Utkal University Bhubaneswar. He is pursuing his research in Computer Science \& Engineering at SOA Deemed-to-be university, Bhubaneswar and had teaching experience of more than two decades. His area of research interests is Mobile ad-hoc networks, Data communication and networking, Computer architecture and Operating systems.

Dr. Meenakshi Sankaran is an alumni of National Institute of Technology Rourkela. She is working as Senior Assistant Professor and also serving as NAAC Program Coordinator of VITBS, VIT Bhopal University. She had 15 years of teaching experience and possesses Research $\&$ Administrative experience of more than a decade. Her research interest mainly focused on Competency Mapping \& Skill development for Generation Z, Educational Psychology, Management Information systems, Information \& Communications Technology, Emotional Intelligence, Mobile Adhoc Networks and Database Management systems.

Dr. Amiya Kumar Rath is a Professor in Computer Science \& Engineering, Burla. At present he is an Senior adviser, ICT for National Assessment \& Accreditation Council (NAAC), India. He contributed over 150 research papers to many national, international journals \& conferences, and author of eight books published by reputed publishers. Earned four awards for contribution to academics and research.
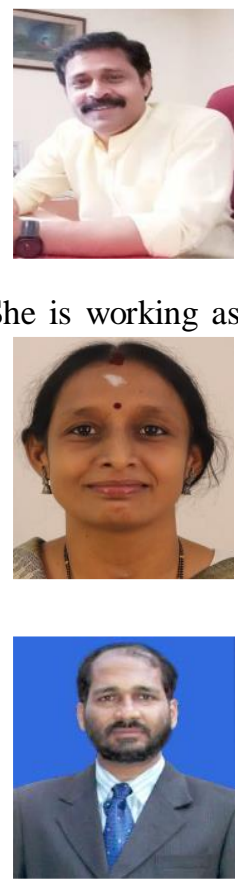